\title{
Situación de los receptores de trasplante de órganos sólidos con COVID-19 en México
}

\author{
COVID-19 in solid organ transplant recipients: \\ national case report from Mexico
}

\author{
José Salvador Aburto-Morales, * José André Madrigal-Bustamante,* \\ Josefina Romero-Méndez, ${ }^{*}$ Cinthya Ayerim Lucio-García* \\ * Centro Nacional de Trasplantes. Ciudad de México, México.
}

\section{RESUMEN}

Introducción: Los pacientes trasplantados que reciben inmunosupresores se encuentran en un mayor riesgo de adquirir COVID-19, presentar complicaciones en su evolución y la concomitante probabilidad de rechazo del órgano trasplantado, además de las dificultades para su prevención, detección oportuna, seguimiento y control, por lo que resulta relevante identificar el estado y evolución de los receptores de trasplante que adquirieron la enfermedad de COVID-19 para el caso mexicano. Material y métodos: Se trata de un estudio transversal, con la información recabada entre el primero de abril y el 25 de mayo de 2020 en México. A partir de fuentes primarias de información, se realizó un análisis descriptivo de frecuencias simples y relativas, como razones, proporciones y tasas, para identificar las características generales de las personas receptoras de trasplante con COVID-19. Resultados: Se reportaron un total de 28 casos confirmados de COVID-19 en personas receptoras de trasplante. De éstos, la mayoría ocurrieron en hombres receptores $(57.14 \%)$ de la Ciudad de México $(78.57 \%)$ y de hospitales de la Secretaría de Salud (46.43\%), mayoritariamente en trasplante renal (92.86\%). El grupo de edad más afectado fue el de 45 a 64 años. Del total de casos, ocho fallecieron, con una letalidad estimada de $28.6 \%$. Conclusiones: Los datos pueden sugerir que la enfermedad por COVID-19 en receptores de trasplante de órganos sólidos suele tener una evolución más severa comparada con la población mexicana en general. La información generada es una herramienta de apoyo para los profesionales y los integrantes de SNDT, para conocer el abordaje de esta epidemia y para comprender más su comportamiento en esta población.

Palabras clave: COVID-19, SARS-CoV-2, pandemias, trasplante, obtención de tejidos y órganos, México.

\begin{abstract}
Introduction: Transplanted patients who receive immunosuppressants are at a higher risk of getting COVID-19 infection, of presenting complications in its evolution and the concomitant probability of rejection of the transplanted organ, besides the difficulties for its prevention, prompt detection, monitoring and control. It is relevant to identify the status and evolution of transplant recipients who acquired COVID-19 disease in the Mexican case. Material and methods: It is a cross-sectional study, for which information was collected between April 1 and May 25, 2020 in Mexico. A descriptive analysis of simple and relative frequencies as ratios, proportions and rates was performed from primary sources of information, to identify the general characteristics of the recipients of transplantation with COVID-19. Results: 28 confirmed cases of COVID-19 were reported in transplant recipients. Of these, the majority occurred in men (57.14\%), in recipients from Mexico City (78.57\%), in facilities from the Ministry of Health (46.43\%) and corresponded to kidney transplantation (92.86\%). The most affected age group was 4564 years. Of the total cases, eight died, it has been estimated a case fatality of $28.6 \%$. Conclusions: The data may suggest that COVID-19 disease in solid organ transplant recipients could have a more severe course compared to the general Mexican population. The information generated is a relevant tool for professionals and members of SNDT to approach to this epidemic and to have a better understand about its pattern.
\end{abstract}

Keywords: COVID-19, SARS-CoV-2, pandemics, transplantation, tissue and organ procurement, Mexico. 


\section{INTRODUCCIÓN}

El nuevo virus SARS-CoV-2 -asociado con una de las más importantes pandemias de las que se tenga registro a lo largo de la historia de la humanidad- se ha diseminado por todo el mundo. Los casos de esta enfermedad, a nivel mundial al mes de mayo, ascienden a los $5,990,613$, con 365,133 muertes y $2,633,440$ pacientes recuperados. ${ }^{1}$

En México, a partir del 28 de febrero se registraron los primeros casos y, para el 29 de mayo de 2020, se diagnosticaron por PCR 81,400 casos; de los cuales, 9,044 fallecieron. ${ }^{2}$ Los datos utilizados para fines de este reporte (al 25 de mayo) muestran un crecimiento acelerado, con 71,105 casos acumulados, 7,633 defunciones y una tasa de letalidad de $10.7 \%$, la cual contrasta con la tasa mundial de letalidad, que es de $6.3 \%$. Los estados con el mayor número de casos confirmados de pacientes con COVID-19 de mayor a menor son: la Ciudad de México (20,235 casos), el Estado de México $(11,523)$, Baja California $(4,250)$ y Tabasco $(3,236)$. Del total de contagios acumulados, $43 \%$ ha afectado a mujeres y $57 \%$ a hombres; además, la mediana de edad se ubica en los 46 años. De manera similar, las entidades federativas con el mayor número de muertes de mayor a menor son: la Ciudad de México (2,024), el Estado de México (872), Baja California (705), Veracruz (416) y Tabasco (327). Las comorbilidades más frecuentes identificadas en los fallecidos han sido la hipertensión (42.8\%), diabetes (37.6\%) y obesidad (26.7\%); la edad más frecuente de las defunciones es de 60 años, teniendo una mayor inclinación para los varones (68\%).

Los estudios publicados a nivel internacional respecto al comportamiento de la COVID-19 han identificado una mayor afectación en pacientes en edad avanzada, con comorbilidades y en aquellos inmunosuprimidos. ${ }^{3}$ Para aquellos receptores de trasplantes de órganos sólidos, la literatura concuerda en una mayor afectación en este grupo, con mayores tasas de letalidad en comparación con la población general, mayor frecuencia en los hombres y específicamente en receptores de trasplante renal; se ha referido también que entre 20 y $30 \%$ de estos pacientes ha requerido manejo hospitalario. .-9 $^{-1}$

En el contexto de esta emergencia sanitaria, el número de intervenciones de donaciones y trasplantes se ha visto sustancialmente afectado a nivel global. ${ }^{10}$ Si bien algunos programas de donación y trasplantes que operan en países con sistemas de salud basados en la cobertura universal y financiada han conseguido

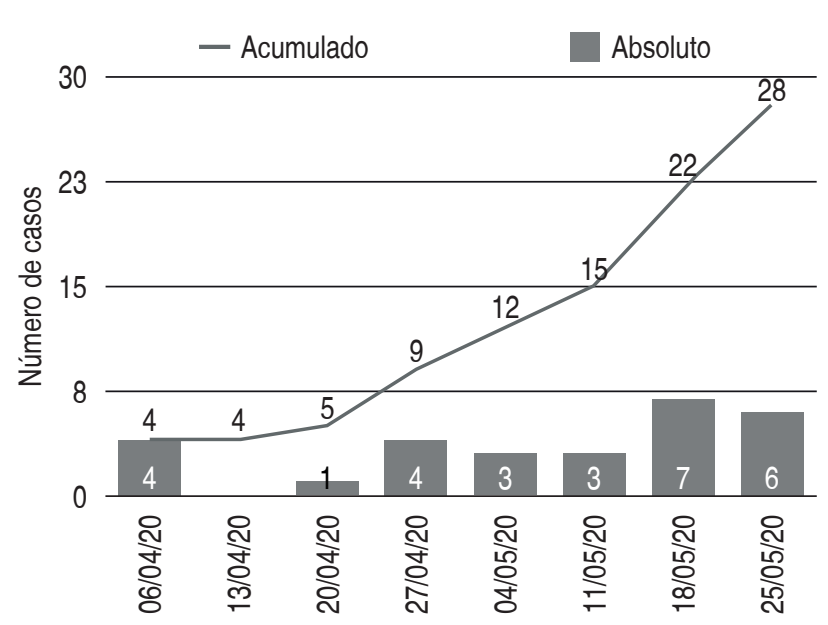

Figura 1: Número de casos confirmados absolutos y acumulados por fecha de reporte en México (2020).

mantenerse abiertos, lo han hecho con limitaciones y con grandes retos debido a la alta transmisión de la enfermedad, la complejidad de la logística requerida y las afectaciones en los ámbitos social, económico y político.

En México, la Secretaría de Salud a través del Centro Nacional de Trasplantes (CENATRA), de manera consistente y coordinada con todos los integrantes del Subsistema Nacional de Trasplantes, emitió las Recomendaciones al Subsistema Nacional de Donación y Trasplantes y su actualización, ${ }^{11}$ la cual considera el aplazamiento hasta nuevo aviso de las actividades de donación y trasplantes de todos los programas a nivel nacional, exceptuando la realización de trasplantes en casos de urgencia nacional (hígado y corazón) y asignación prioritaria (riñón y córnea).

En estas mismas recomendaciones, se incluyó el requerimiento y el formato de reporte para el seguimiento de los receptores de trasplantes infectados por COVID-19 en todo el país. A la fecha, se trabaja en un «Plan de reactivación de los programas de donación y trasplantes a nivel nacional». Además, semanalmente se publica el Boletín informativo especial: trasplantes y COVID-19 como parte del compromiso para el seguimiento de los pacientes receptores de trasplante y por la necesidad de contar con información oportuna y confiable para retroalimentar la toma de decisiones. Éste es un primer reporte del análisis de la información recabada desde el inicio de su operación, el cual comenzó del primero de abril hasta el 25 de mayo de 2020, donde se identificó el estado y evolución de los receptores de trasplante que adquirieron COVID-19 en México. 


\section{MATERIAL Y MÉTODOS}

Se trata de un estudio transversal, con la información recabada entre el primero de abril y el 25 de mayo de 2020 en México. La población de estudio corresponde a los pacientes receptores de trasplante en México que durante dicho periodo adquirieron la enfermedad por SARS-CoV-2. Se incluyó en él a todos los receptores de trasplantes reportados al CENATRA, acorde con la definición operacional de caso confirmado: «persona que cumpla con la definición operacional de caso sospechoso y que cuente con diagnóstico confirmado por la Red Nacional de Laboratorios de Salud Pública reconocidos por el Instituto Nacional de Diagnóstico y Referencia Epidemiológicos (InDRE)». ${ }^{12}$

Los datos recabados de los receptores incluidos se utilizaron exclusivamente con fines de estudio y se manejaron con estricta confidencialidad.

Las fuentes de información fueron primarias, extraídas a partir de formatos específicos para el reporte y seguimiento de casos, establecido en las Recomendaciones al Subsistema Nacional de Donación y Trasplantes, y que los responsables de programa y coordinadores hospitalarios de donación llenaron y enviaron al Centro Nacional de Trasplantes. La información también se sistematiza y publica de manera semanal en el Boletín Informativo Especial: Trasplantes y COVID-19, el cual contiene información actualizada de los receptores de trasplantes y su condición respecto de la infección por SARS-CoV-2 y que, actualmente, se encuentra disponible en el sitio web del Gobierno de México. ${ }^{13}$

Se realizó un análisis descriptivo de frecuencias simples y relativas, como razones, proporciones y tasas, para identificar las características generales de las personas receptoras de trasplante con COVID-19, y por tipo de caso y tipo de trasplante, según órgano, sexo, edad, entidad federativa, institución, desenlace y años transcurridos desde el trasplante al momento de la infección. Este tipo de análisis permite obtener un panorama general de la condición de estos pacientes en México, además de generar nuevas líneas de investigación y acciones particulares en los diferentes programas.

\section{RESULTADOS}

Durante el periodo de estudio, se reportaron un total de 28 casos confirmados de COVID-19 en personas receptoras de trasplante, de los cuales, ocho fallecieron; la letalidad estimada fue de $28.6 \%$.
En la Figura 1 se muestra el número de casos confirmados en valores absolutos y acumulados de acuerdo con la fecha de reporte. El corte se realiza de forma semanal, los días lunes, por lo que la fecha indicada corresponde a la semana inmediata anterior. Se puede observar un discreto incremento en el número de casos reportados en las últimas semanas. En la Tabla 1 se resumen las características generales de los casos confirmados y de aquéllos que tuvieron como desenlace la defunción.

Respecto a los casos confirmados, la mayoría fueron hombres (57.14\%), al igual que en las defunciones (87.5\%), con una razón de H:M de 1.33 y de 7, respectivamente.

El promedio de edad fue mayor en las personas que fallecieron (53.2 años) que en los casos confirmados (48.1 años). Respecto a los grupos de edad, se observó que la mayor frecuencia en los casos confirmados se presentó en el grupo de 45 a 64 años ( $n=$ $14,50 \%)$, tanto para el trasplante renal como para el hepático. Sólo se presentaron cuatro casos (14\%) en pacientes mayores de 65 años.

Sobre el tipo de trasplante, la mayoría de los casos confirmados correspondieron a riñón $(92.86 \%)$; esta diferencia es más notable en las personas que falle-

Tabla 1: Características de los casos confirmados y defunciones por COVID-19 en receptores de trasplante.

\begin{tabular}{lcc}
\hline & $\begin{array}{c}\text { Casos confirmados } \\
(n=28) \\
n(\%)\end{array}$ & $\begin{array}{c}\text { Defunciones } \\
(n=8) \\
n(\%)\end{array}$ \\
Variable & & \\
\hline Sexo & $16(57.14)$ & $7(87.5)$ \\
Masculino & $12(42.86)$ & $1(3.57)$ \\
Femenino & & \\
Edad (años) & 48.14 & 53.25 \\
Promedio & 49 & 56 \\
Mediana & & \\
Tipo de trasplante & $26(92.86)$ & $8(100)$ \\
Riñón & $2(7.14)$ & 0 \\
Hígado & & \\
Entidad federativa & $22(78.57)$ & $6(75.0)$ \\
Ciudad de México & $1(3.57)$ & 0 \\
Coahuila & $3(10.71)$ & $1(12.5)$ \\
Jalisco & $2(7.14)$ & $1(12.5)$ \\
Puebla & & \\
Institución & $11(39.29)$ & $6(75.0)$ \\
IMSS & $13(46.43)$ & $2(25.0)$ \\
SSA & $4(14.29)$ & 0 \\
Privado & 5.84 & 8.93 \\
Promedio de años desde el & & \\
trasplante & & \\
\hline & & \\
\hline
\end{tabular}




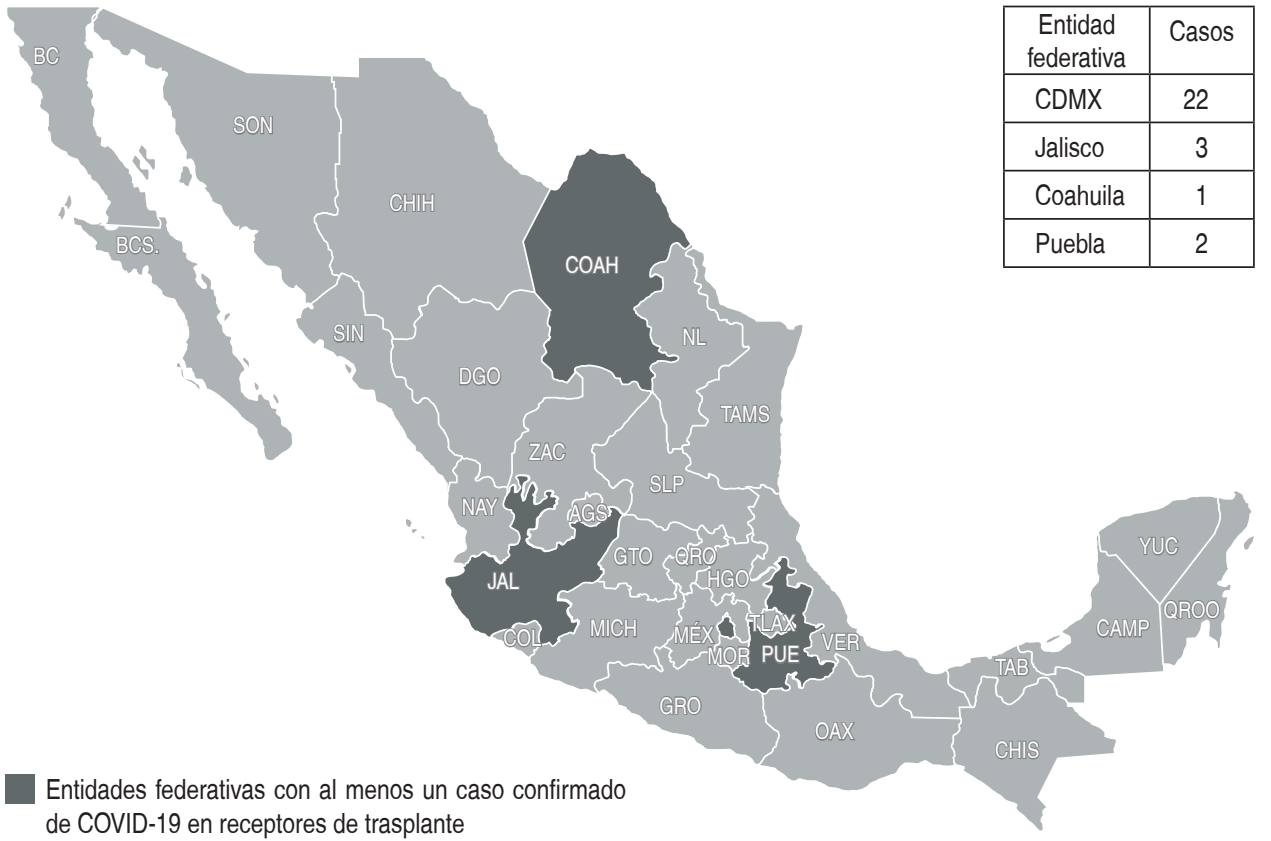

Figura 2:

Entidades federativas con al menos un caso confirmado de COVID-19 en personas receptoras de trasplante en México. cieron, puesto que la totalidad de las defunciones ha sido en personas con trasplante renal. Hasta la fecha, no se recibieron reportes de receptores de trasplante de tejidos que hubieran adquirido COVID-19.

La entidad federativa que hasta el momento registró el mayor número de casos confirmados de COVID-19 en receptores de trasplante fue la Ciudad de México con 22 casos $(79 \%)$, seguida de Jalisco con tres (11\%), Puebla con dos (7\%) y Coahuila con un caso (4\%) (Figura 2).

Respecto de las instituciones, la Secretaría de Salud ha reportado la mayor proporción de casos confirmados (46.43\%), seguida del Instituto Mexicano del Seguro Social $(39.29 \%)$ y los establecimientos privados (14.29\%). Esta relación se invierte en el caso de las personas fallecidas, pues la mayor proporción corresponde al Instituto Mexicano del Seguro Social (75\%) (Figura 3).

Con relación al desenlace, se puede observar que, de la totalidad de casos confirmados, al momento del corte de información, por una parte, la mayoría (nueve casos) estaba hospitalizado; por otra parte, siete casos fueron dados de alta y en cuatro casos se les brindó manejo ambulatorio. De la totalidad de casos confirmados, ocho fenecieron a causa de la enfermedad (Figura 4).

\section{DISCUSIÓN}

$\mathrm{Al}$ igual que los datos generales que se tienen de $\mathrm{CO}$ VID-19 en la población mexicana, los casos y muertes de receptores confirmados se concentran mayormen- te en la Ciudad de México (22 de 28), en la Secretaría de Salud $(46.4 \%$ para trasplantados y en población general el $46.2 \%$ ) y en el IMSS (33.3\% en la población general, y para pacientes trasplantados de $39.3 \%$ ).

De acuerdo con los estudios en otros países revisados, existe una diferencia en cuanto a la mediana de edad reportada en los pacientes trasplantados con COVID-19, ya que para México fue de 49 años, pero para España, Suiza y Nueva York fue mayor a los 55 años. En todos los casos, fue mayor la frecuencia en el sexo masculino (57\% en México; $67 \%$, en España; $57 \%$, en Nueva York, y $67 \%$, en Suiza). Los pacientes con trasplante renal ocupan los primeros lugares

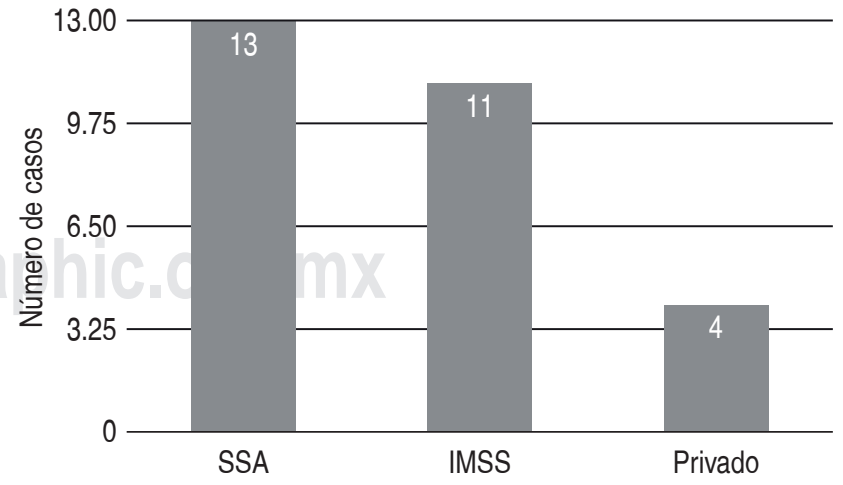

Figura 3: Total de casos confirmados con COVID-19 en personas receptoras de trasplante por institución. 


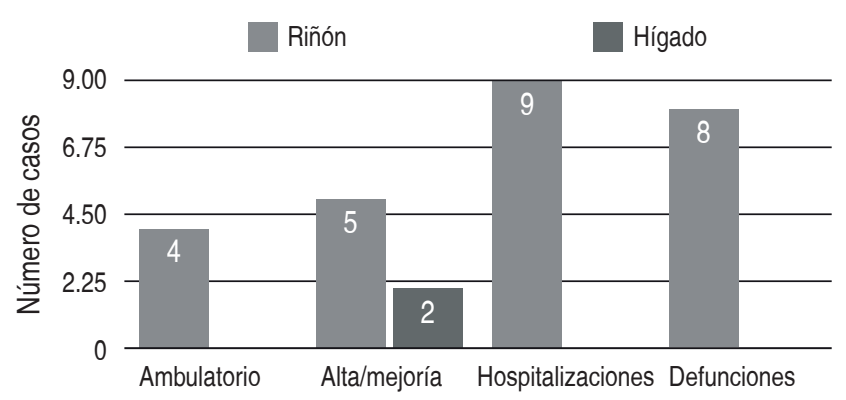

Figura 4: Total de casos confirmados con COVID-19 en personas receptoras de trasplante por tipo de desenlace y tipo de trasplante.

con $93 \%$ para México, $58 \%$ para España y $48 \%$ para Suiza. En segundo lugar, se encuentra el trasplante hepático con $7 \%$ para México y $17 \%$ para España. Cabe señalar que, en general, el riñón es el órgano más trasplantado en los distintos países, lo que podría ser un factor que influya en esta diferencia.

El tiempo transcurrido entre la fecha del trasplante y la presentación de la infección fue de 5.48 años para México; cifra muy similar al caso de España, que reporta cinco años y es menor a los 7.7 años en la serie de casos de Nueva York.

Los reportes de letalidad muestran diferencias que dependen del tiempo de observación y seguimiento de las series de casos, cuyas cifras oscilan desde 18 hasta $30 \%$; una de las más consistentes es el reporte español, que refiere una letalidad total de $20 \%$ en contraste con México, donde la letalidad estimada fue de $28.6 \%$. En el caso mexicano, la tasa se ajustará una vez que se conozca el desenlace de los pacientes que al momento de este reporte se encontraban en hospitalización. Sin embargo, también es notoria la diferencia respecto a la letalidad registrada (10.7\%) en el «Informe técnico diario por coronavirus en México al 25 de mayo de 2020", ${ }^{14}$ lo cual puede sugerir que la enfermedad por SARS-CoV-2 en receptores de trasplante de órganos sólidos suele tener una evolución más severa. No obstante, son necesarios estudios adicionales para comprobar esta posible hipótesis.

Ahora bien, llama también la atención que el porcentaje de fallecimientos en pacientes trasplantados del sexo masculino fue de $87.5 \%$; este porcentaje es mayor comparado con lo reportado para población mexicana, que es de $68.8 \%$.

\section{CONCLUSIONES}

A pesar de que el número de pacientes trasplantados con COVID-19 es pequeño, a la fecha de corte y con
28 casos confirmados, los datos obtenidos son orientadores para conocer el comportamiento de esta infección en la población en estudio. Creemos que, a medida que se cuente con más registros, podremos tener mejores evidencias para generar nuevos estudios y sustentar nuevas recomendaciones en los diferentes programas de donación y trasplantes en Méxi$\mathrm{co}$, a fin de mejorar su detección y control.

La cobertura de este esfuerzo es nacional, pues participan todas las instituciones del sector salud, tanto públicas como privadas, así como de las 32 entidades federativas del país. El Subsistema Nacional de Donación y Trasplantes ha trabajado de manera coordinada desde el inicio de la epidemia en México, lo cual ha permitido tener una visión más amplia de la situación del país y tomar decisiones consensuadas.

Esta estrecha colaboración ha posibilitado la identificación y uso de alternativas de comunicación entre los integrantes del subsistema y los profesionales de la salud en los hospitales, con el objetivo de mejorar la detección y seguimiento de casos. Los programas de trasplantes han definido progresivamente vías de comunicación telefónica o por Internet con los receptores de trasplante para asegurar la vigilancia y seguimiento de cada caso, para que se pueda de esta manera detectar oportunamente los pacientes que estén en riesgo de presentar esta enfermedad.

La información aquí contenida seguramente será de apoyo para los profesionales y los integrantes de SNDT, así como para el abordaje de esta epidemia y para comprender más su comportamiento en esta población.

\section{AGRADECIMIENTOS}

Este trabajo no habría sido posible sin la colaboración de los coordinadores hospitalarios de donación y trasplante en nuestro país, así como de los Centros y Consejos Estatales de Trasplante y Coordinaciones Institucionales. El Centro Nacional de Trasplantes reconoce y agradece su compromiso y trabajo adicional ante la emergencia sanitaria por COVID-19. Son ustedes piedra angular para obtener información periódica de la situación de los pacientes trasplantados con riesgo de COVID-19 y para detonar la detección y el seguimiento oportunos de estos pacientes a fin de evitar consecuencias mayores.

\section{REFERENCIAS}

1. Center for Systems Science and Engineering at Johns Hopkins University. Coronavirus COVID-19 (2019nCoV) [accesado el 4 de junio de 2020]. Disponible en: 
https://www.arcgis.com/apps/opsdashboard/index.html\#/ bda7594740fd40299423467b48e9ecf6

2. Secretaría de Salud. Información Internacional y Nacional sobre el nuevo coronavirus (COVID-2019) [accesado el 4 de junio de 2020]. Disponible en: https://www.gob.mx/salud/documentos/ informacion-internacional-y-nacional-sobre-nuevo-coronavirus2019-ncov

3. Worldometer. Coronavirus cases. Worldometer. 2020; 1-22. doi: 10.1101/2020.01.23.20018549V2.

4. Por E, Coll E, Gayoso J, Ramón Martínez J, Domínguez-Gil B. Ministerio de Sanidad. Organización Nacional de Trasplantes. 2018.

5. Fernández-Ruiz M, Andrés $A$, Loinaz $C$ et al. COVID-19 in solid organ transplant recipients: a single-center case series from Spain. Am J Transplant. 2020. doi: 10.1111/ajt.15929.

6. Montagud-Marrahi E, Cofan F, Torregrosa JV et al. Preliminary data on outcomes of SARS-CoV-2 infection in a Spanish single center cohort of kidney recipients. Am J Transplant. 2020.

7. Tschopp J, L'Huillier A, Mombelli M et al. First experience of SARS-CoV-2 infections in solid organ transplant recipients in the swiss transplant cohort study. Am J Transplant. 2020. doi: 10.1111/ajt.16062.

8. Pereira MR, Mohan S, Cohen DJ et al. COVID-19 in solid organ transplant recipients: Initial report from the US epicenter. Am J Transplant. 2020. doi: 10.1111/ajt.15941.

9. Nair V, Jandovitz N, Hirsch JS et al. COVID-19 in kidney transplant recipients. Am J Transplant. 2020. doi: 10.1111/ ajt.15967.
10. Loupy A, Aubert O, Reese PP, Bastien O, Bayer F, Jacquelinet C. Organ procurement and transplantation during the COVID-19 pandemic. Lancet. 2020; 395 (10237): e95-e96. doi: 10.1016/ s0140-6736(20)31040-0.

11. Centro Nacional de Trasplantes. Recomendaciones al subsistema nacional de donación y trasplantes sobre la infección asociada al SARS-CoV-2 (COVID-19) [accesado el 4 de junio de 2020]. Disponible en: www.gob.mx/cenatra.

12. Lineamiento Estandarizado para la Vigilancia Epidemiológica y por Laboratorio de la enfermedad respiratoria viral. Secretaría de Salud [accesado el 4 de junio de 2020]. Disponible en: https:// www.gob.mx/salud/documentos/lineamiento-estandarizadopara-la-vigilancia-epidemiologica-y-por-laboratorio-de-laenfermedad-respiratoria-viral

13. Boletín informativo especial: trasplante y COVID-19 [accesado el 4 de junio de 2020]. Disponible en: https://www.gob.mx/cenatra/ es/documentos/boletin-informativo-especial-trasplantes-ycovid-19? state=published

14. YouTube. Informe diario por coronavirus en México, 25 de mayo de 2020. [accesado el 4 de junio de 2020]. Disponible en: https:// www.youtube.com/watch?v=TooizgeCPss

Correspondencia:

Cinthya Ayerim Lucio-García

Carretera Picacho-Ajusco Núm. 154,

Col. Jardines en la Montaña, Tlalpan, 14210,

Ciudad de México.

Teléfono: 54879902, ext. 51410

E-mail: cinthya.lucio@salud.gob.mx 